\title{
O modelo SASIS: Validação da simulação da irrigação por sulco
}

\author{
Roberto V. Pordeus ${ }^{1}$, Carlos A. V. de Azevedo², José Dantas Neto², Vera L. A. de Lima², \\ Márcia R. de Q. A. Azevedo ${ }^{3}$ \& José de A. de Matos ${ }^{1}$
}

\begin{abstract}
RESUMO
Nesta pesquisa se objetivou validar a simulação da irrigação por sulco, realizada pelo modelo SASIS através do procedimento matemático de ondas cinemáticas. Utilizam-se, na validação do modelo SASIS, 7 conjuntos de dados que representam diferentes condições de campo, em que o comprimento de sulco variou de 67 a $403 \mathrm{~m}$, a declividade de 0,0016 a $0,0173 \mathrm{~m} \mathrm{~m}^{-1}$, a vazão de 1,1 a 2,0 $\mathrm{L} \mathrm{s}^{-1}$ e o tipo de textura do solo de franco-arenoso a franco silte-argiloso. Para maior consistência da validação, as simulações realizadas por este modelo foram comparadas com as do modelo SIRMOD, além das comparações com as medições de campo. Para a maioria dos dados de campo estudados, as simulações da fase de avanço pelo modelo SASIS mostraram discrepâncias no tempo de avanço, no final da área, inferiores àquelas dadas pelo modelo SIRMOD, em relação às medições de campo. Novamente, para grande parte das condições de campo analisadas as discrepâncias entre os valores simulados pelo modelo SASIS e os medidos em campo do tempo de avanço no final da área, foram pequenas, a ponto de não comprometerem o prognóstico de parâmetros de dimensionamento, avaliação e manejo dos sistemas de irrigação por sulco.
\end{abstract}

Palavras-chave: irrigação superficial, modelo de ondas cinemáticas, manejo de água

\section{The SASIS model: Validation of furrow irrigation simulation}

\begin{abstract}
The aim of this research was to validate the simulation of furrow irrigation performed by the SASIS model through the mathematical procedure of kinematic waves. In the validation of the SASIS model, 7 data sets representing different field conditions were used, in which the furrow length varied from 67 to $403 \mathrm{~m}$, the field slope from 0.0016 to $0.0173 \mathrm{~m} \mathrm{~m}^{-1}$, the inflow from 1.1 to $2.0 \mathrm{~L} \mathrm{~s}^{-1}$ and the soil texture type from sandy loam to silty clay loam. In order to give more consistency to the validation, the simulations accomplished by this model were compared with those from the SIRMOD model, and also compared with data from the field measurements. For most field data studied, the simulations of the advance phase by SASIS model presented discrepancies in the advance time at the end of the area, being inferior to the ones given by SIRMOD model, in relation to field measurements. Again, for the greater part of field conditions analyzed, the discrepancies between the values simulated by SASIS model and those measured in field of the advance time at the end of the area were small, to the point of not compromising the prognostic of design, evaluation and management parameters of furrow irrigation systems.
\end{abstract}

Key words: surface irrigation, kinematic wave model, water management

1 DCA/UFERSA, BR 110, km 47, Costa e Silva, CEP 59625-900, Mossoró, RN. Fone: (84) 3315-1739. Email: rpordeus@ufersa.edu.br

2 UAEAg/UFCG, Av. Aprígio Veloso 882, Bodocongó, CEP 58109-970, Campina Grande, PB. Fone: (83) 3310-1056; (83) 3310-1373; (83) 3310-1349.

Email: cazevedo@deag.ufcg.edu.br; zedantas@deag.ufcg.edu.br; antunes@deag.ufcg.edu.br

3 UEPB, CEP 58109-753, Campina Grande, PB. Fone: (83) 3333-3860. Email: marciarqaa@ibest.com.br 


\section{INTRODUÇÃO}

A simulação matemática da irrigação por superfície tem processos complexos na hidráulica do escoamento superficial, os quais têm sido simulados por modelos computacionais com amplo grau de complexidade e precisão: (Strelkoff \& Katopodes, 1977; Elliott et al., 1982; Walker \& Humpherys, 1983; Strelkoff \& Souza, 1984; Rayej \& Wallender, 1985; Walker \& Skogerboe, 1987; Azevedo, 1992). Tais modelos simulam as fases de avanço, armazenamento, depleção e recessão da água ao longo da superfície do solo e o volume de água infiltrado, escoado e percolado.

O modelo hidrodinâmico resolve completamente as equações de Saint-Venant (equação da continuidade e do movimento), isto é, sem assumir aproximações simplificativas; o modelo de zero inércia baseia-se também nessas equações negligenciando, porém, na equação do movimento, os termos inerciais; já o modelo de ondas cinemáticas se baseia apenas na equação da continuidade e na equação de Manning. Esses três modelos são baseados na solução numérica das equações diferenciais da conservação de massa e de energia e, segundo vários estudos, predizem mais adequadamente o processo da irrigação por superfície que o modelo do balanço de volume de água, Raghuwanshi \& Wallender (1996).

O modelo hidrodinâmico é o mais preciso dos modelos, razão por que é considerado padrão, porém os modelos zero inércia e ondas cinemáticas, apesar de serem mais simples apresentam, em termos gerais, precisão satisfatória sendo, portanto, boas alternativas na simulação da irrigação por superfície (Strelkoff \& Katopodes, 1977; Rayej \& Wallender, 1985; Azevedo, 1992; Valiantzas, 1999).

Os diferentes modelos de simulação da irrigação por superfície foram desenvolvidos para simular um evento de irrigação isolado, assumindo-se que não existe variabilidade espacial nos parâmetros de campo (infiltração, rugosidade, declividade e seção transversal de fluxo); na prática, tem-se verificado a validade desta hipótese, tendo em vista que as simulações se têm aproximado bastante das medições de campo das fases, porém as variabilidades temporais nesses parâmetros são sempre levadas em conta, uma vez que, para a avaliação de qualquer evento de irrigação ao longo da estação de cultivo, novas medições dos parâmetros de campo são realizadas.

Muitos aspectos de uma irrigação por sulco devem ser considerados na qualidade de sua simulação, dentre os quais se citam o modelo matemático empregado e o grau de precisão dos dados de campo, como mais importantes. A qualidade da simulação reflete na precisão do prognóstico de parâmetros extremamente relevantes no dimensionamento, manejo e desempenho da irrigação por sulco, tais como: volumes infiltrados, escoados e percolados, e parâmetros de desempenho da irrigação, que são eficiência de aplicação d'água, perdas d‘água por percolação e escoamento, eficiência de armazenamento e uniformidade de distribuição.

Objetivou-se, com esta pesquisa, validar a simulação da irrigação por sulco com fluxo contínuo realizada pelo modelo matemático computacional SASIS - Software Aplicado à Simulação da Irrigação por Superfície.

\section{MATERIAL E MÉTODOS}

\section{Equações do modelo de ondas cinemáticas}

No modelo matemático de ondas cinemáticas, utilizado nesta pesquisa, se assume que não existe variação da altura de fluxo com a distância, isto é, $\partial y / \partial x=0$, negligenciando-se completamente a equação do movimento sem a qual nada pode ser dito com respeito à dinâmica da forma do perfil superficial de fluxo, ficando a equação da continuidade indeterminada no termo $\partial \mathrm{A} / \partial \mathrm{t}$; para solucionar este problema, ressalta-se haver uma relação única que descreve vazão em função da área de fluxo, substitui-se, então, a equação do movimento pela equação de Manning. O escoamento, estudado apenas cinematicamente, assemelha-se à propagação de uma onda cinemática que entra em colisão, razão por que este modelo é designado ondas cinemáticas. A designação de modelo de escoamento uniforme, também usada, se deve, como é óbvio, à fundamentação da equação de Manning, que descreve o fluxo uniforme.

Assim sendo, as equações que constituem o modelo de ondas cinemáticas, são:

Equação da continuidade:

$$
\frac{\partial \mathrm{A}}{\partial \mathrm{t}}+\frac{\partial \mathrm{Q}}{\partial \mathrm{x}}+\frac{\partial \mathrm{Z}}{\partial \tau}=0
$$

Equação de Manning:

$$
\mathrm{A}=\sqrt{\frac{\mathrm{Q}^{2} \mathrm{n}^{2}}{\mathrm{~S}_{\mathrm{o}} \mathrm{R}^{4 / 3}}}
$$

em que:

$$
\begin{aligned}
& \text { A - área da seção transversal de fluxo, } \mathrm{m}^{2} \\
& \mathrm{t} \text { - tempo de ocorrência, min } \\
& \mathrm{x} \text { - distância de avanço da água, } \mathrm{m} \\
& \tau \text { - tempo de oportunidade de infiltração, min } \\
& \mathrm{Z} \text { - volume infiltrado acumulado por unidade de } \\
& \quad \text { comprimento de sulco, } \mathrm{m}^{3} \mathrm{~m}^{-1} \\
& \mathrm{Q} \text { - vazão, } \mathrm{m}^{3} \mathrm{~s}^{-1} \\
& \mathrm{n} \text { - coeficiente de rugosidade de Manning, } \mathrm{m}^{-1 / 3} \mathrm{~s} \\
& \mathrm{~S}_{\mathrm{o}} \text { - declividade do terreno, } \mathrm{m} \mathrm{m}^{-1} \\
& \mathrm{R} \text { - raio hidráulico, } \mathrm{m}
\end{aligned}
$$

A equação de Manning foi utilizada, nesta análise, para gerar relação única entre vazão e seção hidráulica. Elliott et al. (1982) propuseram relações empíricas para a seção hidráulica, dadas por:

$$
y=\sigma_{1} A^{\sigma_{2}}
$$

e

$$
A^{2} R^{1,33}=\rho_{1} A^{\rho_{2}}
$$

donde:

$\mathrm{y}$ - altura de fluxo, $\mathrm{m}$

$\mathrm{A}$ - área da seção transversal de escoamento, $\mathrm{m}^{2}$

$\mathrm{R}$ - raio hidráulico, $\mathrm{m}$

$\sigma_{1}, \sigma_{2}, \rho_{1}$ e $\rho_{2}$ - parâmetros empíricos que dependem da forma do sulco

Os parâmetros geométricos $\sigma_{1}, \sigma_{2}, \rho_{1}$, e $\rho_{2}$, são considerados constantes para um sulco prismático, ou seja, em um 
evento de irrigação não variam ao longo do comprimento do sulco, válidos apenas para um evento de irrigação específico, haja vista que seus valores podem mudar entre uma irrigação a outra.

Esta hipótese garante que as funções potenciais descrevem adequadamente relações entre altura de fluxo, área, largura da superfície livre d’água, raio hidráulico etc; enfim da equação de Manning e se atendendo à Eq. 4, chega-se a:

$$
S_{0}=\frac{Q^{2} n^{2}}{A^{2} R^{1,33}}=\frac{Q^{2} n^{2}}{\rho_{1} A^{\rho_{2}}}
$$

em que: Q é a descarga de fluxo em $\mathrm{m}^{3} \mathrm{~s}^{-1}$; n é o coeficiente de Manning em $\mathrm{m}^{-1 / 3} \mathrm{~s}$ e $\mathrm{S}_{\mathrm{o}}$ é a declividade média do sulco em $\mathrm{m} \mathrm{m}^{-1}$, de onde se tira Q cuja derivada, em conjunto com a equação da infiltração, se substitui na Eq. 1, seguindo-se uma cadeia de transformações que, afinal, compõem o desenvolvimento do modelo (Walker \& Humpherys, 1983).

Com base nas Eqs. 2 e 5, este tipo de modelo não é aplicável a sulcos quando a declividade é muito pequena, ou seja, tende a zero; na realidade, sua precisão diminuirá quando $\mathrm{S}_{\mathrm{o}}$ se aproximar de zero. Strelkoff \& Katopodes (1977) acharam que ele simula melhor as condições de escoamento quanto maior for a declividade longitudinal.

Usando-se a relação da Eq. 3, a equação de Manning se tornará:

$$
\mathrm{Q}=\alpha \mathrm{A}^{\mathrm{m}}
$$

em que:

$$
\begin{aligned}
& \alpha=\frac{\sqrt{\rho_{1} S_{0}}}{n} \\
& m=\frac{\rho_{2}}{2}
\end{aligned}
$$

sendo $\mathrm{n}$ o coeficiente de rugosidade de Manning em $\mathrm{m}^{-1 / 3} \mathrm{~s}$, $\mathrm{S}_{\mathrm{o}}$ a declividade do sulco em $\mathrm{m} \mathrm{m}^{-1}$ e $\rho_{1}$ e $\rho_{2}$ constantes empíricas ajustadas às medições de campo atuais da geometria do sulco; a e m são constantes empíricas.

\section{Solução numérica do modelo}

A solução numérica das equações do modelo de ondas cinemáticas se baseia no conceito do controle de volume constituído de células individuais deformáveis, descrevendo o perfil superficial de fluxo em um evento de irrigação. A deformação mais acentuada do perfil superficial de fluxo ocorre nas regiões de contorno do sistema. A solução dessas equações leva em consideração que a maior deformação das células ocorre na seção de entrada do fluxo ou, ainda, na seção de saída. Quando se baseia na maior deformação na seção de entrada, as células desenvolvem uma velocidade avante definindo, assim, um sistema Lagrangeano, ou seja, com células em movimento, ocorrendo sua expansão tanto ao longo da distância como do tempo, enquanto se a solução das equações é baseada na deformação na seção de saída, as células se tornam estacionárias, estabelecendo um sistema Euleriano no qual a expansão das células ocorre apenas ao longo do tempo.
Para solução numérica espacial das equações do modelo de ondas cinemáticas utilizou-se, nesta pesquisa, o procedimento de integração Euleriana com aproximação de primeira ordem, apresentado por Walker \& Humpherys (1983) e Wallender (1986), o qual resulta em duas equações algébricas mais estáveis e mais fáceis de serem resolvidas em microcomputadores. Conceitualmente, a aproximação considera o perfil superficial e subsuperficial de água ao longo da porção da área umedecida durante etapas seqüenciais de cálculo. A Figura 1 ilustra os perfis superficial e subsuperficial de fluxo nos tempos $t_{\mathrm{i}-1}$ e $\mathrm{t}_{\mathrm{i}}$, identificando as células que os compõem. Durante cada etapa de cálculo o fluxo d’água avança a uma distância incremental, $\delta x$; por exemplo, durante o primeiro intervalo de tempo (primeira etapa de cálculo), se estende a uma distância $\delta \mathrm{x}_{1}$; no segundo intervalo de tempo, a uma distância $\delta x_{2}$, e assim sucessivamente, podendo-se generalizar, para a distância da frente de avanço, $\mathrm{x}_{\mathrm{i}}$, no tempo $\mathrm{t}_{\mathrm{i}}$, da seguinte maneira:

$$
\mathrm{x}_{\mathrm{i}}=\sum_{\mathrm{k}=1}^{\mathrm{i}} \delta \mathrm{x}_{\mathrm{k}}
$$

em que $\delta x_{k}$ é o k-ésimo incremento de espaço, definido pelo avanço durante o intervalo de tempo, quando $\mathrm{i}=\mathrm{k}$, donde $\mathrm{k}$ é número de incrementos de tempo.

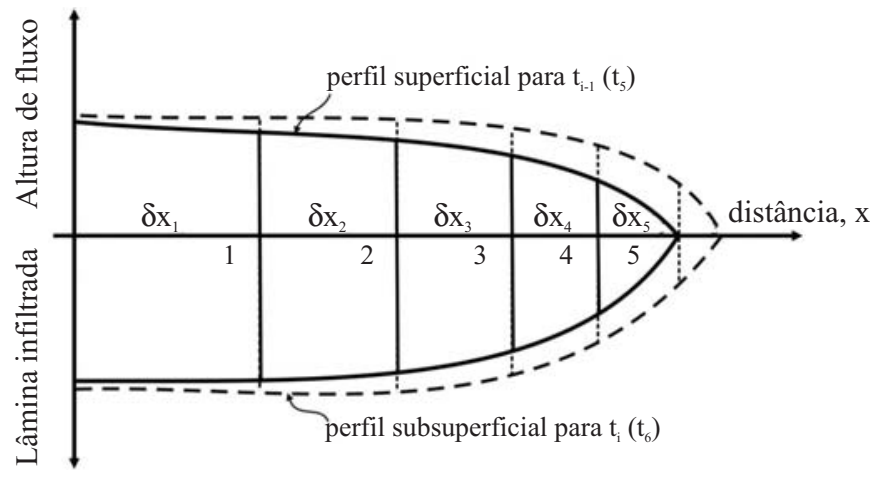

Fonte : Walker \& Skogerboe (1987)

Figura 1. Esquema da progressão do fluxo superficial e subsuperficial (infiltração) para intervalo de tempo constante

Uma célula típica é ilustrada na Figura 2, exibindo os perfis nas etapas de cálculo $\mathrm{t}_{\mathrm{i}-1}$ e $\mathrm{t}_{\mathrm{i}}$. As anotações $\mathrm{J}, \mathrm{M}, \mathrm{L}$ e $\mathrm{R}$ são introduzidas em cada célula para identificar as variáveis que descrevem as condições de fluxo com relação ao tempo e ao espaço. Desta maneira, as variáveis subscritas por $\mathrm{J}$ ou $\mathrm{M}$ se referem às condições de fluxo no tempo $\mathrm{t}_{\mathrm{i}-1}$ e nas fronteiras à esquerda (montante) e à direita (jusante) da célula, respectivamente. Similarmente, L e R são subscritos nas fronteiras à esquerda e à direita da célula no tempo $t_{i}$. Combinando-se as células de todos os incrementos de tempo ter-se-á uma grade de cálculo no plano $(\mathrm{x}, \mathrm{t})$, na qual as trajetórias de avanço e recessão podem ser traçadas (Figura 3). Observa-se, neste plano, que durante a fase de avanço as células são retangulares, exceto as da frente de avanço, que são triangulares por 
não existir fluxo na fronteira à jusante dessas células, nos tempos $t_{i}$ e $t_{i-1}$. Durante as fases de armazenamento e depleção todas as células são retangulares; já durante a recessão, a célula da extremidade à montante é triangular, pelas mesmas razões do comportamento do fluxo na frente de avanço mas as demais células são retangulares. A largura de cada célula é determinada pela distância da frente de avanço durante cada etapa de cálculo $\delta$ t, que se torna, então, uma das incógnitas do problema, uma vez que se considerou $\delta$ t constante; a isto se chama uma solução espacial das equações do modelo de ondas cinemáticas, porque $\delta x$ é uma incógnita, enquanto o valor de $\delta \mathrm{t}$ é definido pelo usuário do modelo.

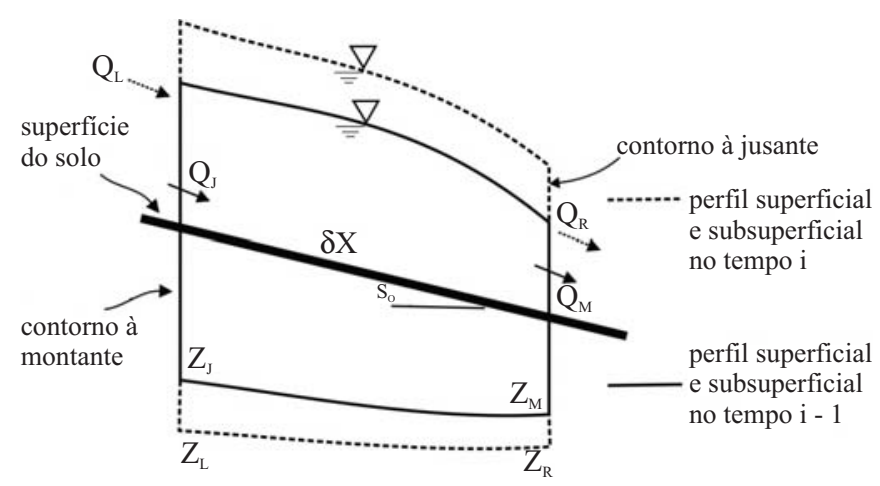

Figura 2. Célula deformada de fluxo (Walker \& Skorgeboe, 1987)

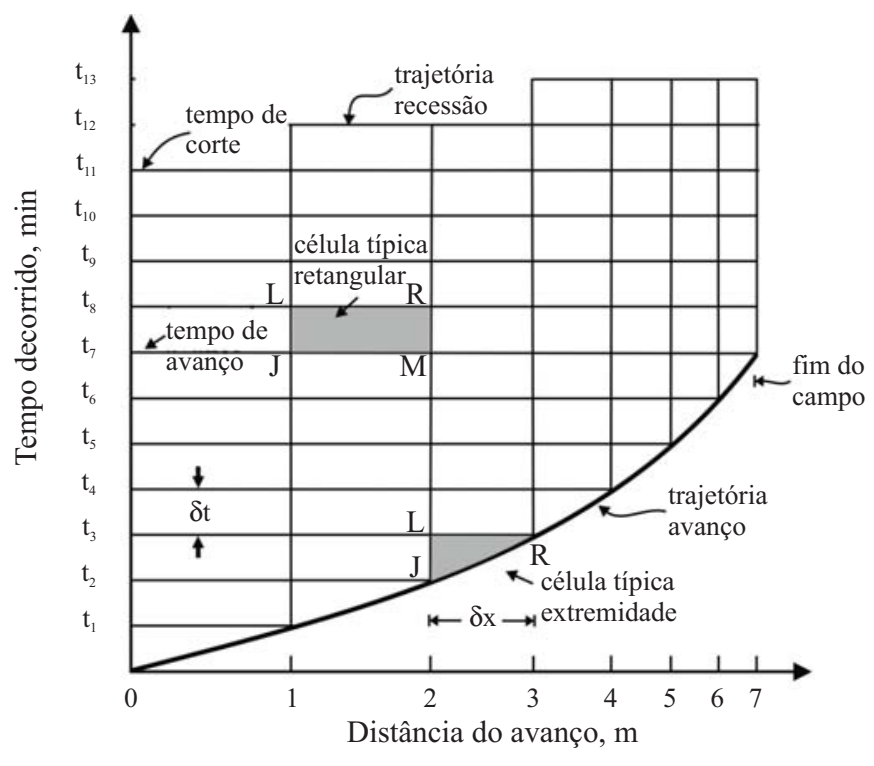

Figura 3. Malha computacional (Walker \& Skogerboe, 1987)

Para se obter a forma integrada da equação da continuidade (Eq. 1), primeiro se integra, analiticamente, com relação à distância e depois numericamente, quanto ao tempo. Tem-se, então:

$\int_{t}^{t+\delta t}\left(\int_{x}^{x+\delta x} \frac{\partial Q}{\partial x} d x\right) d t+\int_{x}^{x+\delta x}\left(\int_{t}^{t+\delta t} \frac{\partial A}{\partial t} d t\right) d x+\int_{x}^{x+\delta x}\left(\int_{t}^{t+\delta t} \frac{\partial Z}{\partial \tau} d t\right) d x=0$

em que a primeira integração resulta em: $\int_{t}^{t+\delta t}[Q(x+\delta x, t)-Q(x, t)] d t+\int_{x}^{x+\delta x}[A(x, t+\delta t)-A(x, t)] d x+$

$\int_{x}^{x+\delta x}[Z(x, t+\delta t)-Z(x, t)] d x=0$

e a segunda em:

$$
\begin{aligned}
& \frac{1}{2}\left[\left(\mathrm{Q}_{x+\delta x, t}-Q_{x, t}\right)_{t+\delta t}+\left(Q_{x+\delta x, t}-Q_{x, t}\right)_{t}\right] \delta t+ \\
& \frac{1}{2}\left[\left(A_{x, t+\delta t}-A_{x, t}\right)_{x+\delta x}+\left(A_{x, t+\delta t}-A_{x, t}\right)_{x}\right] \delta x+ \\
& \frac{1}{2}\left[\left(Z_{x, t+\delta t}-Z_{x, t}\right)_{x+\delta x}+\left(Z_{x, t+\delta t}-Z_{x, t}\right)_{x}\right] \delta x=0
\end{aligned}
$$

Uma vez que durante a fase de avanço o fluxo vai diminuindo ao longo da distância, devido ao efeito da taxa de infiltração d‘água no solo, caindo para zero na frente de avanço, a não linearidade no perfil, tanto superficial como subsuperficial, se torna bastante acentuada fazendo com que o fator $1 / 2$ não seja apropriado para ponderar as condições de fluxo; desta forma, uma ponderação adequada deverá estar entre $1 / 2$ a 1 . Criam-se, então, os fatores $\theta$ e $\phi$ para ponderarem a não linearidade no perfil, respectivamente, com relação ao tempo e ao espaço. Esses fatores são extremamente importantes apenas durante a fase de avanço, porque depois desta fase ocorre tendência de se estabelecer condições estáveis de fluxo. A ponderação deve ser maior no tempo $t+\delta t$ visto que durante a fase de avanço a não linearidade aumenta com o tempo, sendo máxima quando a água atinge o final da área (condições de fluxo nulas); além disso, também deve ser maior na seção de entrada uma vez que a altura de fluxo varia mais rapidamente nesta seção, e é nela que a vazão está sendo aplicada.

Substituindo-se, na Eq. 12, 1/2 por $\theta$, $(1-\theta), \phi,(1-\phi)$ e se escrevendo as variáveis de fluxo em termos das anotações de uma célula computacional (Figura 2), tem-se:

$$
\begin{aligned}
& {\left[\theta\left(Q_{R}-Q_{L}\right)+(1-\theta)\left(Q_{M}-Q_{J}\right)\right] \delta t+} \\
& {\left[\varphi\left(A_{L}-A_{J}\right)+(1-\varphi)\left(A_{R}-A_{M}\right)\right] \delta x+} \\
& {\left[\varphi\left(Z_{L}-Z_{J}\right)+(1-\varphi)\left(Z_{R}-Z_{M}\right)\right] \delta x=0}
\end{aligned}
$$

sendo $\theta$ e $\phi$ coeficientes de ponderação temporal e espacial, respectivamente, cujos valores variam entre $1 / 2$ e 1 ; em geral, tem-se tomado valores iguais a 0,65 e 0,51 para $\theta$ e $\phi$, respectivamente. Walker (2001) em seu software SIRMOD III, usa 0,60 para ambos, isto é, o mesmo valor adotado nesta pesquisa.

A solução numérica é obtida resolvendo-se a Eq. 13 para cada célula na malha computacional (Figura 3) começandose horizontalmente da esquerda para a direita, em cada etapa de cálculo. As únicas incógnitas em cada célula, são $\mathrm{Q}_{\mathrm{R}}$ e $\mathrm{A}_{\mathrm{R}}$; entretanto, uma vez que $Q$ está sendo calculado explicitamente pela equação de Manning (Eq. 6), não se o considera uma incógnita; o mesmo caso é aplicado a Z (infiltração) o qual está sendo calculado pela equação de Kostiakov-Lewis; o que se faz é substituir as equações de $\mathrm{Q}$ e $\mathrm{Z}$ na Eq. 13 ficando esta, então, apenas com uma incógnita $\left(A_{R}\right)$.

Substituindo-se a Eq. 6 na Eq. 13 e se dividindo por $\theta \alpha / \delta$ t, chegar-se-á à seguinte equação não linear em $A_{R}$ : 


$$
\begin{aligned}
& A_{R}^{m}+\left(\frac{1-\varphi}{\theta \alpha}\right) \frac{\delta x}{\delta t} A_{R}-A_{L}^{m}+\left(\frac{1-\theta}{\theta}\right)\left(A_{M}^{m}-A_{J}^{m}\right)+ \\
& \frac{\varphi}{\theta \alpha}\left(A_{L}+Z_{L}-A_{J}-Z_{J}\right) \frac{\delta x}{\delta t}+\left(\frac{1-\varphi}{\theta \alpha}\right)\left(Z_{R}-A_{M}-Z_{M}\right) \frac{\delta x}{\delta t}=0
\end{aligned}
$$

Objetivando-se simplificar a Eq. 14, isolam-se as constantes e variáveis com valores conhecidos a partir da etapa de cálculo anterior nos coeficientes $C_{1}$ e $C_{2}$. Tem-se, então:

$$
\mathrm{C}_{1}=\left(\frac{1-\varphi}{\theta \alpha}\right) \frac{\delta \mathrm{x}}{\delta \mathrm{t}}
$$

e,

$$
\begin{aligned}
& \mathrm{C}_{2}=-\mathrm{A}_{\mathrm{L}}^{\mathrm{m}}-\left(\frac{1-\theta}{\theta}\right) \mathrm{A}_{\mathrm{J}}^{\mathrm{m}+1}+\left(\frac{1-\theta}{\theta}\right) \mathrm{A}_{\mathrm{M}}^{\mathrm{m}+1}+ \\
& \frac{\varphi}{\alpha \theta}\left(\mathrm{A}_{\mathrm{L}}+\mathrm{Z}_{\mathrm{L}}-\mathrm{A}_{\mathrm{J}}-\mathrm{Z}_{\mathrm{J}}\right) \frac{\delta \mathrm{x}}{\delta \mathrm{t}}+\left(\frac{1-\varphi}{\alpha \theta}\right)\left(\mathrm{Z}_{\mathrm{R}}-\mathrm{A}_{\mathrm{M}}-\mathrm{Z}_{\mathrm{M}}\right) \frac{\delta \mathrm{x}}{\delta \mathrm{t}}
\end{aligned}
$$

chegando-se à equação

$$
\mathrm{A}_{\mathrm{R}}^{\mathrm{m}+1}+\mathrm{C}_{1} \mathrm{~A}_{\mathrm{R}}+\mathrm{C}_{2}=0
$$

A Eq. 17 é utilizada para células interiores e para a primeira célula, depois da primeira etapa de cálculo. Uma vez que a Eq. 17 é solucionada implicitamente (pelo método de Newton-Raphson) para cada célula separadamente, não se tem, então, uma matriz. Esta equação é usada de forma implícita para determinar $A_{R}$ e, depois, se determina explicitamente $\mathrm{Q}_{\mathrm{R}}$, pela equação de Manning (Eq. 6)

\section{Condições iniciais}

As Eqs. 15 a 17 geram soluções para todas as células, exceto para aquelas na frente de avanço (célula da extremidade do avanço), em que as condições de contorno resultam em $A_{R}, A_{J}, A_{M}, Z_{R}, Z_{J}$ e $Z_{M}$ iguais a zero. Para essas células, a formulação matemática das Eqs. 15 a 17, se reduz a:

$$
-\mathrm{A}_{\mathrm{L}}^{\mathrm{m}}+\frac{\varphi}{\alpha \theta}\left(\mathrm{A}_{\mathrm{L}}+\mathrm{Z}_{\mathrm{L}}\right) \frac{\delta \mathrm{x}_{1}}{\delta \mathrm{t}}=0
$$

Como $A_{L}$ é conhecida da solução da célula anterior e $Z_{L}$ é função única do tempo de oportunidade de infiltração, a única incógnita nas células da frente de avanço é, então, a distância incremental $(\delta x)$; desta forma, a Eq. 18 se reduz a:

$$
\delta \mathrm{x}_{1}=\frac{\theta \alpha \mathrm{A}_{\mathrm{L}}^{\mathrm{m}} \delta \mathrm{t}}{\varphi\left(\mathrm{A}_{\mathrm{L}}+\mathrm{Z}_{\mathrm{L}}\right)}
$$

Quando o suprimento de água na entrada da área é cortado, assume-se que a área no contorno esquerdo, $A_{L}$, cai imediatamente a zero. A partir do corte da água se inicia a fase de recessão e os cálculos são feitos de forma similar aos da fase de avanço e terminam quando a área molhada é reduzida a um valor igual ou menor que 5\% do valor da área na entrada do sulco.

\section{Condições de contorno}

Os sistemas de irrigação por superfície apresentam duas condições de contorno de jusante: 1) uma célula triangular durante a fase de avanço e 2) uma célula retangular para a fronteira do campo. No caso (2), várias configurações são possíveis mas se tem usualmente, uma condição de $\mathrm{Q}_{\mathrm{N}}=0$ (sulco fechado) ou um fluxo de saída uniforme; entretanto, em algumas condições a descarga pode passar sobre ou através de uma estrutura hidráulica com característica diferente caso em que é necessário se estabelecer uma avaliação da descarga da estrutura para descrever o escoamento na condição de contorno.

O contorno à montante é definido pelas condições de fluxo na cabeceira da área irrigada, na qual $\mathrm{A}=0$ e $\mathrm{Q}=0$ para $\mathrm{t}=0$ e $\mathrm{t}>\mathrm{t}_{\mathrm{ap}}$ (tempo de aplicação da água) e $\mathrm{A}=\mathrm{A}_{0}$ para $0<\mathrm{t} \leq \mathrm{t}_{\mathrm{ap}}$. As condições de fluxo no contorno à jusante são descritas pelas Eqs. 6 e 19, respectivamente, para as fases de avanço e armazenamento, com ocorrência de escoamento superficial. Quando o fluxo atinge o final do campo, as variáveis $\mathrm{M}$ e $\mathrm{R}$ não serão zero para todos os intervalos de tempo, para cujas condições, como pode ser generalizado para a drenagem livre, dique, ou condições controladas, a condição de contorno pode ser descrita pela equação de Manning (Eq. 6).

Dentre as fases da irrigação superficial simuladas o avanço é a mais importante, em virtude de contribuir, de forma mais decisiva, para as variações no tempo de oportunidade de infiltração principalmente na irrigação por sulco, o que resulta na desuniformidade da lâmina infiltrada; assim sendo, o modelo SASIS faz a simulação apenas da fase de avanço, considerando o tempo de depleção constante ao longo da área e igual ao tempo de corte do fluxo d'água no sulco, hipótese que leva em conta o fato de que na irrigação por sulco a duração da fase de recessão é muito curta, em razão não apenas da declividade da superfície do solo mas, também, do volume de água armazenado no sulco, no momento de corte da vazão, que é muito pequeno comparado ao de uma bacia ou de uma faixa, não contribuindo significativamente para o volume de água infiltrado.

Os dados de campo utilizados na validação do modelo SASIS corresponderam a quatro conjuntos de dados (PISG1, PISG2, PISG3 e PISG4) coletados nesta pesquisa, referentes às avaliações de campo de eventos de irrigação por sulco no Perímetro Irrigado de São Gonçalo, no município de Sousa, PB, publicados por Azevedo et al. (2001) e Pordeus et al. (2003); dois conjuntos de dados (AMALGACQ, propriedade privada, e GUFCQ, fazenda da Utah State University em Logan, USA) publicados por Azevedo (1992), empregados na demonstração do modelo SIRTOM, e um conjunto de dados (KWF-Kimberly Wheel Furrow) publicados por Walker \& Skogerboe (1987). Esses conjuntos de dados (Tabela 1) representam diferentes condições de campo, em que o comprimento de sulco variou de 67 a $403 \mathrm{~m}$, a declividade de 0,0016 a $0,0173 \mathrm{~m} \mathrm{~m}^{-1}$, a vazão de 1,1 a $2,0 \mathrm{~L} \mathrm{~s}^{-1}$ e o tipo de textura do solo de franco arenoso a franco silte-argiloso, e dados de avanço medido em campo (Tabela 2). Para maior consistência da validação do modelo SASIS, as simulações realizadas por este modelo foram comparadas com as do modelo SIRMOD (Walker, 1989), além das comparações com as medições de campo. 
Tabela 1. Dados de campo utilizados na validação do modelo SASIS

\begin{tabular}{|c|c|c|c|c|c|c|c|}
\hline Dados de Campo & PISG1 & PISG2 & PISG3 & PISG4 & KWF & AMALGACQ & GUFCQ \\
\hline Tipo de Solo & $\begin{array}{l}\text { Franco argilo } \\
\text { arenoso }\end{array}$ & $\begin{array}{l}\text { Franco argilo } \\
\text { arenoso }\end{array}$ & Franco arenoso & $\begin{array}{l}\text { Franco argilo } \\
\text { arenoso }\end{array}$ & $\begin{array}{l}\text { Franco silte } \\
\text { argiloso }\end{array}$ & Silte argiloso & Silte arenoso \\
\hline Vazão $\left(\mathrm{L} \mathrm{s}^{-1}\right)^{\star}$ & 1,33 & 1,47 & 1,54 & 1,13 & 1,50 & 1,80 & 1,30 \\
\hline Comprimento do sulco (m) & 67 & 84 & 70 & 115 & 360 & 403 & 217 \\
\hline Declividade $\left(\mathrm{m} \mathrm{m}^{-1}\right)$ & 0,0030 & 0,0016 & 0,0043 & 0,0024 & 0,0104 & 0,0066 & 0,0173 \\
\hline Coeficiente de Manning, $n\left(\mathrm{~m}^{-1 / 3} \mathrm{~s}\right)$ & 0,020 & 0,020 & 0,025 & 0,020 & 0,013 & 0,013 & 0,013 \\
\hline Parâmetro da Seção, $\rho_{1}$ & 0,291 & 0,185 & 0,532 & 0,339 & 0,730 & 0,730 & 0,730 \\
\hline Parâmetro da Seção, $\rho_{2}$ & 2,847 & 2,766 & 2,840 & 2,806 & 2,980 & 2,980 & 2,980 \\
\hline$k\left(m^{3} m^{-a} m^{-1}\right)$ & 0,03781 & 0,02931 & 0,01024 & 0,0054 & 0,0088 & 0,00182 & 0,00896 \\
\hline $\mathrm{a}$ & 0,165 & 0,302 & 0,326 & 0,412 & 0,533 & 0,234 & 0,0 \\
\hline$f_{0}\left(m^{3} m^{-1} m^{-1}\right)$ & 0,000186 & 0,000186 & 0,000264 & 0,000186 & 0,00017 & 0,00019 & 0,000022 \\
\hline$Z_{\mathrm{re}}(\mathrm{m})$ & 0,090 & 0,060 & 0,020 & 0,020 & 0,090 & 0,090 & 0,050 \\
\hline
\end{tabular}

PISG1, PISG2, PISG3 e PISG4 - dados de campo obtidos no Perímetro Irrigado de São Gonçalo, PB; KWF: dados de campo publicados por Walker \& Skogerboe (1987); AMALGACQ e GUFCQ: dados de campo publicados por Azevedo (1992); *Vazão praticada pelo irrigante

Tabela 2. Dados de avanço d'água medidos em campo e utilizados na validação do modelo SASIS

\begin{tabular}{|c|c|c|c|c|c|c|c|c|c|c|c|c|c|}
\hline \multicolumn{2}{|c|}{ PISG1 } & \multicolumn{2}{|c|}{ PISG2 } & \multicolumn{2}{|c|}{ PISG3 } & \multicolumn{2}{|c|}{ PISG4 } & \multicolumn{2}{|c|}{ KWF } & \multicolumn{2}{|c|}{ AMALGACQ } & \multicolumn{2}{|c|}{ GUFCQ } \\
\hline$X A(m)$ & TA (min) & XA (m) & TA (min) & $X A(m)$ & TA (min) & $X A(m)$ & TA (min) & $X A(m)$ & TA (min) & $X A(m)$ & TA (min) & $X A(m)$ & TA (min) \\
\hline 0 & 0 & 0 & 0 & 0 & 0 & 0 & 0 & 0 & 0 & 0 & 0 & 0 & 0 \\
\hline 6,7 & 2 & 9,09 & 1,05 & 7 & 1 & 11,5 & 3 & 40 & 5 & 31 & 12 & 31 & 4 \\
\hline 13,4 & 4 & 18,18 & 2,35 & 14 & 2 & 23 & 5 & 80 & 14 & 62 & 22 & 62 & 8 \\
\hline 20,1 & 6 & 27,27 & 3,6 & 21 & 3 & 34,5 & 7 & 100 & 20 & 93 & 30 & 93 & 12 \\
\hline 26,8 & 9 & 36,36 & 5,0 & 28 & 5 & 46 & 10 & 120 & 30 & 124 & 46 & 124 & 16 \\
\hline 33,5 & 13 & 45,45 & 6,5 & 35 & 7 & 57,5 & 14 & 140 & 37 & 155 & 53 & 155 & 20 \\
\hline 40,2 & 16 & 54,54 & 8,5 & 42 & 10 & 69 & 17 & 160 & 48 & 186 & 68 & 186 & 24 \\
\hline 46,9 & 20 & 63,64 & 9,65 & 49 & 13 & 80,5 & 27 & 200 & 75 & 217 & 85 & 217 & 28 \\
\hline 53,6 & 23 & 72,73 & 11,55 & 56 & 16 & 92 & 40 & 220 & 89 & 248 & 98 & & \\
\hline 60,3 & 27 & 81,82 & 13,6 & 63 & 19 & 103,5 & 48 & 240 & 102 & 279 & 120 & & \\
\hline 67 & 32 & 90,91 & 15,65 & 70 & 24 & 115 & 66 & 275 & 130 & 310 & 140 & & \\
\hline & & 100 & 17,95 & & & & & 300 & 150 & 341 & 155 & & \\
\hline & & & & & & & & 320 & 170 & 372 & 191 & & \\
\hline & & & & & & & & 350 & 200 & 403 & 232 & & \\
\hline & & & & & & & & 360 & 208 & & & & \\
\hline
\end{tabular}

PISG1, PISG2, PISG3 e PISG4 - dados de campo obtidos no Perímetro Irrigado de São Gonçalo, PB; KWF: dados de campo publicados por Walker \& Skogerboe (1987); AMALGACQ e GUFCQ: dados de campo publicados por Azevedo (1992); *Vazão praticada pelo irrigante; ** Vazão determinada em projeto, pelos autores; XA- distância de avanço d'água medida em campo; TA- tempo de avanço

\section{RESULTADOS E DISCUSSÃO}

As curvas de avanço correspondentes às medições de campo e simulações pelos modelos SIRMOD e SASIS para a irrigação por sulco com fluxo contínuo, são apresentadas na Figura 4. Observa-se semelhança quando se comparam as curvas simuladas por ambos os modelos com as medições de campo. Na maioria das simulações, o tempo de avanço é superestimado pelos dois modelos. Para os dados de campo PISG1 observa-se, na Tabela 3 que, no final da área, o tempo de avanço é superestimado em $8 \mathrm{~min}$, pelo modelo SASIS, e em 35,2 min pelo SIRMOD correspondendo, portanto, a uma variação de 20 e $110 \%$, respectivamente; verifica-se, na Figura 4A, que o avanço simulado pelo SASIS ao longo da área se aproxima mais dos valores medidos em campo que o SIRMOD; já a curva de avanço simulada pelo modelo SIRMOD se acerca mais dos valores medidos em campo, apenas nos primeiros $50 \%$ do comprimento do sulco; no restante, a curva de avanço se torna menos linear em relação à simulada pelo SASIS e aos valores medidos em campo, à medida que se avizinha do final da área; os valores simulados pelo SA-
SIS se aproximam dos valores medidos em campo do início da área até o seu final, o que não ocorre com o SIRMOD, o qual simulou 27,2 min a mais no final da área que o SASIS; para este caso, verifica-se melhor simulação do tempo de avanço pelo modelo SASIS.

Observa-se, no exemplo PISG2 e na Figura 4B, que mais uma vez os valores simulados pelo modelo SASIS se aproximam mais dos valores medidos em campo que o SIRMOD;

Tabela 3. Tempo de avanço no final da área medido em campo e simulado pelos modelos SIRMOD e SASIS

\begin{tabular}{|c|c|c|c|c|c|}
\hline \multirow{2}{*}{$\begin{array}{l}\text { Dados } \\
\text { de Campo }\end{array}$} & \multirow{2}{*}{ Campo } & \multicolumn{2}{|c|}{ SIRMOD } & \multicolumn{2}{|c|}{ SASIS } \\
\hline & & Simulado & $\Delta(\%)$ & Simulado & $\Delta(\%)$ \\
\hline PISG1 & 32 & 67,2 & +100 & 40 & +20 \\
\hline PISG2 & 69 & 113,6 & $+64,6$ & 65 & $-5,8$ \\
\hline PISG3 & 24 & 23,4 & $-2,5$ & 30 & +25 \\
\hline PISG4 & 66 & 56,6 & $-14,2$ & 65 & $-1,5$ \\
\hline KWF & 200 & 190 & -5 & 130 & -35 \\
\hline AMALGACQ & 232 & 67,3 & -71 & 265 & $+14,2$ \\
\hline GUFCQ & 28 & 57,3 & $+104,6$ & 45 & $+60,7$ \\
\hline
\end{tabular}

$\Delta$ (\%) - Diferença percentual em relação aos valores medidos em campo 

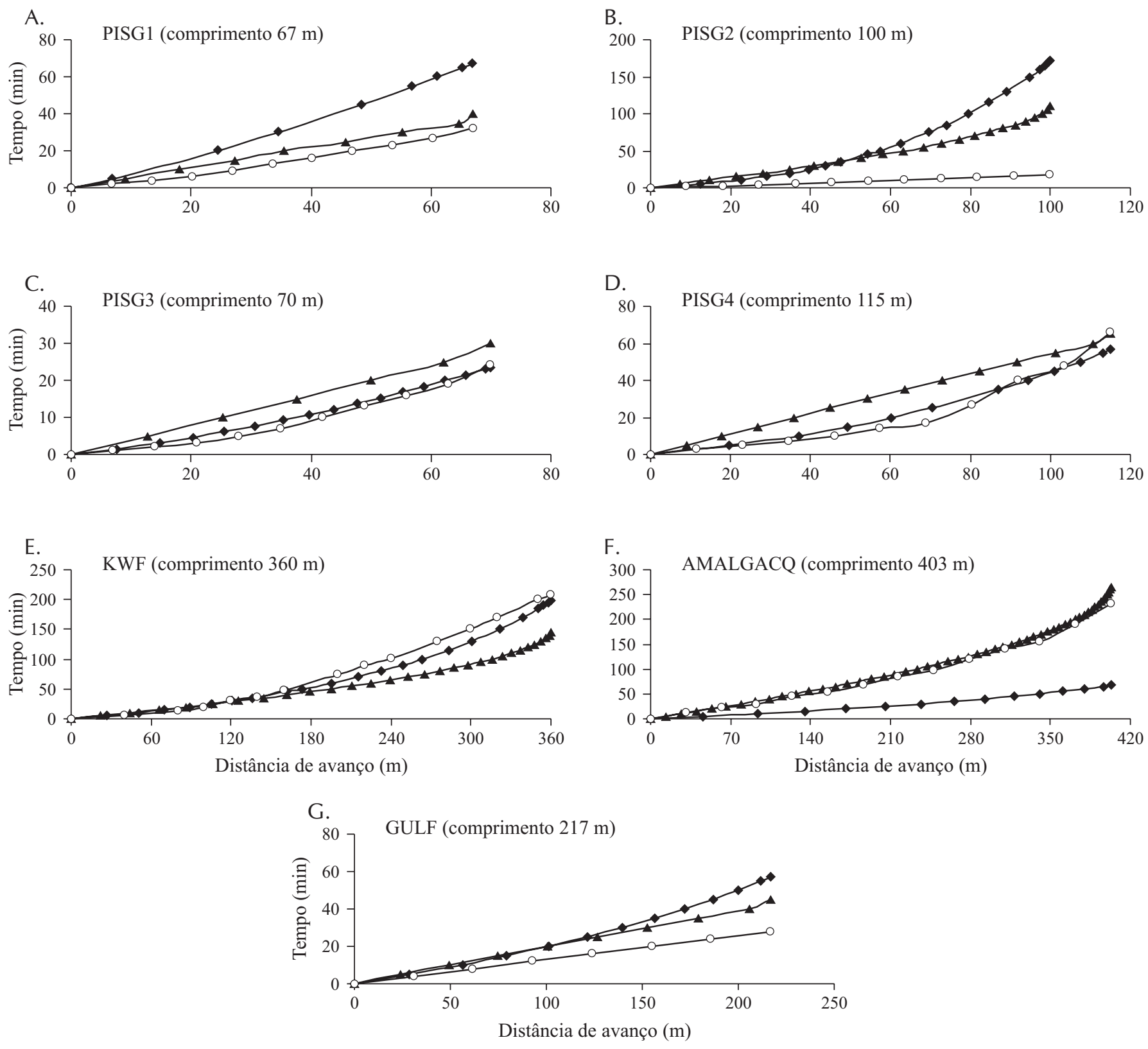

$\multimap$ Avanço campo $\rightarrow$ Avanço SIRMOD $\quad \leftarrow$ Avanço SASIS

Figura 4. Curvas de avanço medidas em campo e simuladas pelos modelos SIRMOD e SASIS

o tempo de avanço no final da área é subestimado pelo SASIS em 5,8\% e superestimado pelo SIRMOD em 64,6\%; os tempos de avanço medidos em campo e simulados por esses modelos foram, respectivamente, de 69,65 e 113,6 min (Tabela 3); a curva de avanço simulada pelo SIRMOD apresenta o mesmo comportamento do exemplo anterior, isto é, nos primeiros 50\% do comprimento do sulco os valores simulados se aproximam mais dos valores medidos com valores mais próximos no início da área, enquanto no restante da área a diferença é crescente sempre que se aproxima do final do campo.

Para os dados de campo PISG3 (Figura 4C), quando se comparam os valores de avanço medidos com os simulados vê-se que o SIRMOD se aproxima mais dos dados de campo que o SASIS; o SIRMOD subestimou o tempo de avanço no final da área, em 2,5\%, enquanto o SASIS o superestima em $25 \%$; para este exemplo, os tempos de avanço medido e simulado pelo SIRMOD e SASIS no final da área, foram de 24,23,4 e 30 min (Tabela 3) respectivamente e, embora a diferença percentual entre os tempos de avanço medido e simulado pelo SASIS tenha sido de $25 \%$, a diferença absoluta foi de apenas 6 min e a diferença entre o tempo simulado pelos dois modelos foi de apenas 6,6 min, o que mostra consistência na simulação realizada pelo modelo SASIS.

Observa-se, na Figura 4D, para os dados de campo PISG4, que o avanço simulado pelo SIRMOD se acerca mais dos valores de avanço medidos que o SASIS, que superestimou o avanço praticamente em todo o comprimento 
do sulco, com valores maiores em torno do centro da área, o que ocorreu também com o SIRMOD, porém com menores diferenças; nesta mesma figura ainda se constata que, exatamente nesta região, ocorre mudança no comportamento da curva de avanço medido em campo mas, no final da área, o tempo de avanço simulado pelo SASIS se assemelha mais ao de campo; para este exemplo, os tempos de avanço medido no final da área e simulado pelos modelos SASIS e SIRMOD foram, respectivamente, de 66,65 e 56,6 min (Tabela 3); conclui-se, daí, que o SASIS subestimou o avanço em apenas $1 \mathrm{~min}$, enquanto o SIRMOD em 9,4 min, correspondendo a variações percentuais de 1,5 e $14,2 \%$, respectivamente, em relação aos dados de campo. Embora o SIRMOD tenha apresentado variação maior no final da área, o avanço simulado ao longo da área se assemelhou mais aos valores medidos, resultando em um prognóstico do perfil de infiltração mais coerente com o que aconteceu em campo.

No exemplo KWF o tempo de avanço medido no final da área foi de 200 min e os simulados pelos modelos SASIS e SIRMOD foram, respectivamente, 130 e $190 \mathrm{~min}$ constatando-se, então, que o modelo SASIS subestimou o avanço em 35\% e o SIRMOD em 5\% (Tabela 3); verificase, na Figura 4E, que nos primeiros $50 \%$ do comprimento da área os avanços simulados pelos dois modelos se assemelham aos medidos em campo, subestimando-os no restante da área, com maiores diferenças sempre que se aproxima do final da área.

Quanto aos dados de campo AMALGACQ nota-se, na Figura 4F, que o avanço simulado pelo modelo SASIS é similar, ao longo de toda a área, ao medido em campo, o que não ocorre com o avanço simulado pelo modelo SIRMOD; o tempo de avanço medido no final da área e o simulado pelos modelos SASIS e SIRMOD foi, respectivamente, 232, 265 e 67,3 min (Tabela 3), verificando-se que o modelo SASIS superestimou o avanço em $33 \mathrm{~min}$, enquanto o SIRMOD o subestimou em 164,7 min, correspondendo a variações percentuais de 14,2 e 71\%, respectivamente; desta forma, o modelo SASIS apresentou maior precisão na simulação.

Observa-se, para os dados GUFCQ, que na Figura 4G, as curvas de avanço obtidas pelos modelos SASIS e SIRMOD se assemelham, no geral, à curva de avanço medida em campo; entretanto, o tempo de avanço medido no final da área e simulado pelos modelos SASIS e SIRMOD, foi 28, 45 e 57,3 min (Tabela 3), respectivamente, apresentando superestimativas de 17 (SASIS) e 29,3 min (SIRMOD), que se referem a variações percentuais de 60,7 e 104,6\%. O modelo SIRMOD o superestimou em $43,9 \%$ a mais que o SASIS.

Na maioria dos exemplos, os tempos de avanço simulados se assemelham mais aos valores medidos em campo nos primeiros $50 \%$ do comprimento do sulco porque, provavelmente, a partir desse trecho o avanço se vai tornando mais lento, devido ao efeito da infiltração d'água na velocidade do fluxo que diminuída ao longo do sulco, aumenta o grau de não linearidade na curva de avanço. É bem mais fácil simular uma reta que uma curva. No restante da área a dife- rença entre os valores simulados e os medidos cresce sempre que se aproxima do final da área e, no geral, os valores simulados pelos dois modelos não apresentam grandes diferenças com relação aos medidos em campo.

De acordo com Levien (2003), o avanço é muito mais rápido no início que no final do sulco, em virtude da infiltração contribuir para redução na velocidade de avanço da água ao longo do sulco; segundo o autor, a uniformidade de irrigação depende da uniformidade do tempo de oportunidade de infiltração da água nos diferentes pontos da parcela irrigada; por sua vez, esta uniformidade é maior quanto mais rápido for o tempo de avanço.

Constatou-se, ante o exposto, que em cinco exemplos (PISG1, PISG2, PISG4, AMALGACQ e GUFCQ), o tempo de avanço no final da área simulado pelo SASIS se aproximou mais dos valores medidos em campo contra dois exemplos (PISG3 e KWF) simulados pelo SIRMOD. Entre os exemplos estudados a maior diferença percentual entre o tempo de avanço medido e o simulado no final da área (Tabela 3) ocorreu para o exemplo GUFCQ que, pelo SASIS, foi de 60,7\% e, pelo SIRMOD, de 104,6\% .

A diferença entre os dados observados e os simulados pelos modelos (SASIS e SIRMOD) depende decisivamente do grau de precisão e representatividade dos parâmetros de entrada requeridos (seção transversal, declividade, rugosidade e parâmetros da equação de infiltração d’água no solo), conseguido quando da realização dos ensaios de campo.

Os resultados apresentados na Tabela 3 e na Figura 4 constatam a viabilidade da aplicação do modelo desenvolvido (SASIS) nesta pesquisa para simulação e racionalização da irrigação por sulco com fluxo contínuo, uma vez que as simulações se assemelham bastante aos valores medidos em campo e, ainda, o referido modelo apresenta grande facilidade de operação, possuindo integração total ao Windows em todas as suas versões, permitindo ao usuário não apenas a entrada de dados de maneira clara e prática mas, também, mudanças em alguns parâmetros do modelo, ajustando-se à realidade de campo em questão. Existem alguns módulos no software SASIS que utilizam as simulações na determinação de parâmetros de dimensionamento, avaliação e manejo da irrigação por sulco, a exemplo do módulo que investiga a vazão ótima, isto é, aquela que minimiza as perdas de água por percolação e escoamento.

\section{CONCLUSÕES}

1. Para a maioria dos dados de campo estudados as simulações da fase de avanço pelo modelo SASIS apresentaram discrepâncias no tempo de avanço no final da área, inferiores às identificadas pelo modelo SIRMOD, em relação às medições de campo.

2. Novamente, para grande parte das condições de campo analisadas as discrepâncias entre os valores simulados pelo modelo SASIS e medidos em campo no tempo de avanço no final da área foram pequenas, a ponto de não comprometerem o prognóstico de parâmetros de dimensionamento, avaliação e manejo dos sistemas de irrigação por sulco. 


\section{AGRADECIMENTOS}

Os autores agradecem ao CNPq, pelo apoio financeiro ao trabalho.

\section{LITERATURA CITADA}

Azevedo, C. A. V. de. Real-time solution of the inverse furrow advance problem. Agricultural and irrigation engineering department, Logon: Utah State University, 263p. 1992. Ph.D. Dissertation

Azevedo, C. A. V. de; Pordeus, R. V.; Lima, V. L. A. de; Dantas Neto, J. Behavior of the optimal discharge in furrow discharge in furrow irrigation with continuous flow. ASAE Annual International Meeting, Sacramento, Calinornia, paper n. 012165, 2001, p.1-13.

Elliott, R. L.; Walker, W. R.; Skogerboe, G. V. Zero inertia modeling of furrow irrigation advance. Journal of the Irrigation and Drainage Division, ASCE, v.108(IR3), p-179195, 1982.

Levien, S. L. A. Irrigação por superfície: Sistemas de irrigação por superfície. Mossoró: ESAM, 2003. 20p.

Pordeus, R. V.; Azevedo, C. A. V. de; Lima, V. L. A. de; Dantas Neto, J. Field evaluation of water infiltration profile in opened and blocked furrow irrigation with continuous flow. ASAE Annual International Meeting, Las Vegas, paper $n$. 032244, 2003, p.1-12.
Raghuwanshi, N.; Wallender, W. W. Modeling of seasonal furrow irrigation. Journal of the Irrigation and Drainage Engineering, ASCE v.122, n.4, p.235-242. 1996.

Rayej, M.; Wallender, W. W. Furrow Irrigation Simulation Time Reduction. Journal of the Irrigation and Drainage Division, ASCE, v.111, n.2, p.134-146, 1985.

Strelkoff, T.; Katopodes, N. D. End Depth Under Zero-Inertia Conditions. Journal of the Hydraulics Division. ASCE, 103(HY1), p.699-611, 1977.

Strelkoff, T.; Souza, F. Modeling effect of depth on furrow infiltration. Journal of Irrigation and Drainage Engineering. ASCE. v.110, n.4, p.375-387, 1984.

Valiantzas, J. D. Explicit time of advance formula for furrow desing. Journal of Irrigationand Drainage Engineering. Davis, v.125, n.1, p.19-25, 1999.

Walker, W. R. Sirmod, Surface irrigation simulation software. Logan: Utah State University. 1989.

Walker, W. R. Software for simulation, design, and evaluation of surface irrigation - User's guide and technical documentation. Logan: Utah State University, Biological and Irrigation Engineering Department, 2001. 63p.

Walker, W. R.; Humpherys, A. S. Kinematic-wave furrow irrigation model. Journal of the Irrigation and Drainage Division, ASCE, New York, 109(IR4), p.377-392, 1983.

Walker, W. R.; Skogerboe, G. V. Surface irrigation: Theory and practice. 1.ed. New Jersey: Prentice-Hall, Inc., Englewood Cliffs, 1987, 470p.

Wallender, W. W. Furrow model with spatially varying infiltration. Transactions of the ASAE, St. Joseph, v.29, n.4, p.1012-1016, 1986. 